\title{
Output Feedback Active Suspension Control With Higher Order Terminal Sliding Mode
}

\author{
Jagat Jyoti Rath, Student Member, IEEE, Michael Defoort, Hamid Reza Karimi, Senior Member, IEEE, \\ and Kalyana Chakravarthy Veluvolu, Senior Member, IEEE
}

\begin{abstract}
The control of an automotive suspension system using hydraulic actuators is a highly complex nonlinear control task dealing with system nonlinearities, external disturbances, and uncertainties. In this work, an output feedback active suspension control scheme is proposed to achieve a ride comfort while maintaining the road holding for the vehicle. To design the controller, the states of the nonlinear system are first estimated using a highgain observer where the suspension stroke is the only measurable output. The controller is then designed using a recursive derivative nonsingular higher order terminal sliding mode approach that avoids singularity. The practical stability for the closed-loop observer-controller pair is established. Simulation results for the quarter-wheel vehicle over various road conditions demonstrate the effectiveness of the proposed control in improving the suspension performance in both the time and frequency domains.
\end{abstract}

Index Terms-Active suspension control, higher order sliding mode, output feedback, terminal sliding mode.

\section{INTRODUCTION}

A CTIVE suspension control [1], [2] has been a widely implemented control procedure, leading to significant developments in improving the passenger comfort while maintaining good road holding capabilities for commercial vehicles [3]-[5].

Manuscript received February 20, 2016; revised July 16, 2016; accepted August 13, 2016. Date of publication September 20, 2016; date of current version January 10, 2017. This research was supported by the Basic Science Research Program through the National Research Foundation of Korea (NRF) funded by the Ministry of Education, Science and Technology under the Grant NRF-2014R1A1A2A10056145 and in part by the BK21 Plus project funded by the Ministry of Education, Korea (21A20131600011). (Corresponding author: Kalyana Chakravarthy Veluvolu.)

J. J. Rath is with the School of Electronics Engineering, College of IT Engineering, Kyungpook National University, Daegu 702-701, South Korea (e-mail: jagat.rath@ knu.ac.kr).

M. Defoort is with the LAMIH, CNRS UMR 8201, University of Valenciennes, 59314 Valenciennes, France (e-mail: mdefoort@univvalenciennes.fr).

$\mathrm{H}$. R. Karimi is with the Department of Mechanical Engineering, Politecnico di Milano, 20156 Milan, Italy (e-mail: hamidreza.karimi@ polimi.it).

K. C. Veluvolu is with the School of Electronics Engineering, College of IT Engineering, Kyungpook National University, Daegu 702-701, South Korea, and also with the School of Mechanical and Aerospace Engineering, Nanyang Technological University, Singapore 639798 (e-mail: veluvolu@ee.knu.ac.kr).
Apart from improving the ride quality, suspension control is also employed to maintain vehicle stability, avoid rollover conditions, etc. [6]. Various robust control approaches such as backstepping control [2], [7], [8], sliding mode control [9], [10], $H_{\infty}$ control [1], [11], fuzzy control [12], etc., have been deployed to provide active suspension control. The analysis of the suspension performance under the influence of such controllers has been one of the most complex and interesting nonlinear control problems in automotive vehicle research.

The advancement in active suspension control has led to the inclusion of various actuators such as hydraulic [3], [4], electromagnetic [5], etc., for providing effective control over various road conditions and driving scenarios. Hydraulic actuators involve severe nonlinear dynamics, but have been widely implemented due to their small bandwidth, reliability, and ease of control [13]. In [11], two active control strategies based on the model reference adaptive control and $H_{\infty}$ control were proposed for a quarter car suspension equipped with hydraulic actuators. Similarly, in [7] and [2], robust $H_{\infty}$ based control approaches were proposed for full vehicle suspension systems controlled using hydraulic actuators. The use of sliding mode control for a suspension system actuated by a hydraulic actuator is discussed in [9].

The backstepping technique [14]-[16] has been established as one of the significant approaches widely employed for suspension control. Various works have considered the backstepping approach [2], [7], [8] for the design of robust controllers for active suspension control. In [8], a saturated adaptive robust control approach was proposed for active control of a halfcar nonlinear suspension system affected by road bumps in the presence of uncertainties and actuator saturation. These works, consider state feedback based approaches where, it is generally assumed that the suspension forces are measured while the road inputs act as exogenous inputs. Overcoming this assumption, robust output feedback approaches were proposed in [1] and [9] to provide active control of the suspension system. In [1], a dynamic $H_{\infty}$ controller was employed while in [9] a sliding mode controller was employed to deal with the active suspension control problem. Sliding mode based controllers [17]-[19] and observers [20]-[24] have been employed for robust performance of dynamic systems in the presence of disturbances and uncertainties. Sliding mode control as a robust control strategy for active suspension control has been widely analyzed in the works of [10], [25] and [9] amongst others. In [25], a proportionalintegral (PI) sliding mode control scheme was proposed for the active control of a linear quarter car suspension system. In [10], 
an adaptive sliding mode control was proposed for a nonlinear active suspension system using a Takagi-Sugeno (T-S) fuzzy approach. Recently, in [27] a nonlinear extended state observer based approach was designed for the control of hydraulic actuators. Employing this concept, a high gain observer (HGO) based integral sliding mode control, designed using the backstepping approach was discussed in [30]. The previous works on backstepping-based design for pure feedback systems such as the active suspension system [9], [10], [25], employed firstorder sliding mode (FOSM) controllers. The FOSM controllers are subject to chattering and necessitate the use of low-pass filters. The problem of singularity in the controller design as a result of the backstepping design procedure restricts the use of higher order sliding mode (HOSM) controllers such as the super-twisting algorithm (STA) [18], [19]. In this paper, a recursive structure is developed to avoid the singularity problem in the controller design and it also facilitates the use of HOSM to further improve the robustness properties. In the previous works on output feedback control for active suspension systems, multiple outputs such as tire deflection [1], sprung and unsprung mass displacements, [25], etc., were considered. However, in this study, we consider the suspension stroke which is easily measurable as the only output. The necessity of providing a robust output feedback suspension control, in the presence of the system nonlinearities and exogenous road inputs while ensuring practical stability, forms the focus of the work proposed in this paper.

In this work, we present a robust output feedback active suspension control approach for the nonlinear quarter car suspension system actuated by nonlinear hydraulic actuators. To develop the observer-based control, we initially formulate the nonlinear suspension and actuator dynamics. The developed model is affected by spring, damper, and actuator nonlinearities and exogenous road inputs in the form of various road profiles and road defects. To estimate the states [26] of the nonlinear system an HGO [27]-[29] is employed, which establishes practical bounded stability of the error dynamics under the assumptions of bounded exogenous road inputs. Employing these estimated states, a robust HOSM controller based on STA [31], [32] is then proposed. Further, a recursive derivative integral structure [33] based on a nonsingular terminal sliding mode (NSTSM) procedure is formulated to develop the controllers. Under this procedure, an NSTSM-integral sliding surface is designed for the quarter vehicle system. The contributions of this work can be listed as follows.

1) The HGO for the suspension-hydraulic actuator system affected by exogenous road inputs in the presence of suspension spring, damper, and actuator nonlinearities is developed. The practical stability of the estimation error is established.

2) An output feedback controller, employing the recursive derivative NSTSM, is proposed. This procedure, similar to the backstepping technique, ensures the ease of design and robustness.

3) The singularity problem in the design of the nonlinear controller is avoided and an HOSM controller based on STA is developed to reduce the chattering phenomenon.

4) Closed-loop stability of the observer-controller pair is established to ensure practical bounded stability.
Employing the designed output feedback controller, the performance of the controller is evaluated for various scenarios such as road conditions, driving velocities, parametric uncertainties, and sensor noise. The performance of the active control in enhancing passenger comfort while maintaining good road holding is demonstrated through extensive simulations conducted on the quarter vehicle model.

\section{A. Dynamics: Suspension and Actuator}

The suspension system of a vehicle is responsible for ensuring passenger comfort while maintaining the vehicle tires on the ground. The dynamics of the suspension system are governed by the motion of the vehicle mass, i.e., the sprung mass and the tire body along with the suspension mass, henceforth, referred to as the unsprung mass. The unsprung mass under the influence of exogenous road inputs generates continuous excitations that are damped out by the suspension unit to ensure passenger comfort. The dynamics of the sprung and unsprung masses for a quarter vehicle model can be given as [34], [35]

$$
\left\{\begin{array}{l}
m_{s} \ddot{z}_{s}=-k_{s} z_{\mathrm{su}}-b_{s} \dot{z}_{\mathrm{su}}-\varphi+U_{s} \\
m_{u} \ddot{z}_{u}=k_{s} z_{\mathrm{su}}+b_{s} \dot{z}_{\mathrm{su}}+\varphi-U_{s}-f_{u}\left(z_{u}, t\right)
\end{array}\right.
$$

where $m_{s}$ is the sprung mass, $m_{u}$ is the unsprung mass, $k_{s}$ is the spring stiffness, $b_{s}$ is the spring damping constant, $\varphi$ is suspension nonlinearities, $f_{u}$ is the exogenous road input, and $U_{s}$ is the active controlled force to be provided by the hydraulic actuator. The suspension deflection, i.e., stroke is defined as

$$
z_{\mathrm{su}}=z_{s}-z_{u}
$$

where $z_{s}$ and $z_{u}$ are the sprung mass and unsprung mass displacements, respectively. The nonlinearity in the suspension dynamics is given as [35]

$$
\varphi=k_{n} z_{\mathrm{su}}^{3}+b_{n} \sqrt{\left|\dot{z}_{\mathrm{su}}\right|} \operatorname{sign}\left(\dot{z}_{\mathrm{su}}\right)
$$

where $k_{n}$ and $b_{n}$ denote the nonlinear coefficients of the spring and damper units of the suspension system, respectively. The road input affecting the unsprung mass can be modeled as a function of the tire deflection $z_{u \xi}$ as [36]

$$
f_{u}\left(z_{u}, t\right)=m_{u}\left(k_{t} z_{u \xi}+b_{t} \dot{z}_{u \xi}\right)
$$

where $k_{t}$ and $b_{t}$ are the tire stiffness and damping, respectively. The tire deflection $z_{u \xi}$ is given as $z_{u \xi}=z_{u}-\xi(t)$, where $\xi$ constitutes the road inputs. The road input responsible for continuous excitation of the suspension unit can be given as $\xi=z_{r}+z_{\mathrm{def}}$, where $z_{r}$ is the road roughness profile [1], [35] determined on the basis of the ISO standards given as

$$
\dot{\xi}(t)=-2 \pi n_{o} v_{x} \xi(t)+2 \pi \sqrt{\sigma_{r} v_{x}} w_{o}
$$

where $v_{x}$ is the longitudinal velocity in $\mathrm{m} / s, \sigma_{r}$ is the road roughness coefficient for different road classes, $n_{o}$ is the reference space frequency, and $w_{o}$ is a white Gaussian noise with a zero mean. The road defects $z_{\text {def }}$ correspond to road conditions, such as bumps, potholes, etc., act as shocks to the suspension system, and are often modeled as sinusoidal inputs [1], [2]. The dynamics of the hydraulic actuator necessary to provide the 
active control $U_{s}$ can be given as [27], [34]

$$
\dot{U}_{s}=\lambda U \sqrt{P_{s}-\operatorname{sign}(U) \frac{U_{s}}{A_{p}}}-A_{p}^{2} \alpha \dot{z}_{\mathrm{su}}-\frac{C_{d}}{A_{p}} U_{s}
$$

where $\lambda=\frac{A_{p} C_{d} w \alpha}{\sqrt{\rho}}, P_{s}$ is the supply pressure of fluid, $A_{p}$ is the piston area, $C_{d}$ is the discharge coefficient, $w$ is the valve width, $\alpha$ is a function of the bulk modulus of the fluid, and $U$ is the spool valve input that can be the controlled voltage or current.

\section{B. Active Control Objectives}

The performance objectives of the active suspension control are twofold: ensuring increased passenger comfort and maintaining road holding. Passenger comfort is achieved by the reduction in the acceleration of the sprung mass. Similarly, road holding refers to the ability of the suspension control to keep the wheel and consequently the vehicle on the ground through the reduction of tire deflection. To achieve these control objectives, the following system requirements are complied with:

1) Road holding must be maintained at all times for the active suspension control to ensure ride safety. To ensure road holding, the root mean square (RMS) value of the wheel load is bounded by

$$
\max \left(\left\|f_{u}\right\|\right) \leq \frac{\left(m_{s}+m_{u}\right) g}{3}
$$

where $\|\cdot\|$ refers to the 2-norm and $g$ is the gravity acceleration. This bound of the dynamic wheel load requires that its maximum value is less than the static wheel load, to ensure that the wheel remains on the ground [37].

2) The maximum possible suspension stroke is fixed to $\pm 0.05 \mathrm{~m}$ to ensure practical feasibility. Similarly, the maximum active force is bounded by $\pm 1000 \mathrm{~N}$ with its peak magnitude at $\pm 2500 \mathrm{~N}$.

The reduction in RMS amplitudes of $\ddot{z}_{s}$ and $z_{u \xi}$ over time in comparison with the passive suspension can be expressed as a measure of the efficacy of an active suspension control scheme. With the above-mentioned system requirements complied with, the performance of the suspension system is then analyzed by the following performance measure:

$$
P_{\text {obj }}=1-\frac{\left\|\mathrm{obj}_{\text {act }}\right\|_{\text {RMS }}}{\left\|\mathrm{obj}_{\text {pas }}\right\|_{\text {RMS }}}
$$

where obj refers to the entity measured, i.e., suspension deflection, sprung mass acceleration or tire deflection. The active measure is denoted by objact while the passive measure is denoted by obj $j_{\text {pas }}$. The improvement in passenger comfort and road holding capabilities can be then determined by a positive increase in their respective performance measures. The active control of a suspension system must also be analyzed from its frequency domain perspective. Specifically it was discussed in [13], that to improve the passenger comfort, the applied active suspension control must account for motion sickness $(0-1 \mathrm{~Hz})$ and head toss $(2-8 \mathrm{~Hz})$. It has been established by the analysis of various suspension configurations that the range of interest for passenger comfort and road holding is between 0 and $10 \mathrm{~Hz}[13]$.
In order to achieve the above-mentioned objectives, we design an observer-based output feedback controller to reduce the sprung mass acceleration and suspension deflection while ensuring good road holding for the vehicle. The efficiency of the designed controller is analyzed by the performance measure (8) for ensuring that the time domain objectives are complied with. Further, the improvement of the frequency response of the actively controlled system is also shown.

\section{Closed-loop Observer-Controller}

In this section, we propose an HGO to estimate the states of the nonlinear system and then design an NSTSM controller employing the estimated states to achieve the control objectives. To develop the observer-controller approach, a nonlinear model integrating the suspension dynamics (1) and the actuator dynamics (6) is formulated as follows:

$$
\left\{\begin{array}{l}
\dot{x}=A x+G(x, U) U+\Phi(x, t)+\Theta(t) \\
y=C x
\end{array}\right.
$$

where the states of the system are

$$
x=\left[\begin{array}{lll}
x_{1} & x_{2} & x_{3}
\end{array}\right]^{T}=\left[\begin{array}{lll}
z_{\mathrm{su}} & \dot{z}_{\mathrm{su}} & M U_{s}
\end{array}\right]^{T}
$$

where the superscript " $\mathrm{T}$ " stands for the matrix transpose. The system matrices are given as

$$
\begin{gathered}
A=\left[\begin{array}{lll}
0 & 1 & 0 \\
0 & 0 & 1 \\
0 & 0 & 0
\end{array}\right], G(x, U)=\left[\begin{array}{c}
0 \\
0 \\
M g\left(x_{3}, U\right)
\end{array}\right], \\
\Phi(x, t)=\left[\begin{array}{lll}
0 & \varphi_{a} & \varphi_{b}
\end{array}\right]^{T}, \Theta(t)=\left[\begin{array}{lll}
0 & f_{u} & 0
\end{array}\right]^{T}
\end{gathered}
$$

with $M=\frac{m_{s}+m_{u}}{m_{s} m_{u}}, g\left(x_{3}, U\right)=\lambda \sqrt{\frac{P_{s}-\operatorname{sign}(U) \frac{x_{3}}{M A_{p}}}{\rho}}$, $\varphi_{a}=-M \varphi-k_{s} M x_{1}-b_{s} M x_{2}, \varphi_{b}=-M A_{p}^{2} \alpha x_{2}-\frac{C_{t}}{A_{p}} x_{3}$. The output matrix is given as

$$
C=\left[\begin{array}{lll}
1 & 0 & 0
\end{array}\right] \text {. }
$$

The displacement/velocity measurements for the suspension system in practical scenarios are generally relative measurements such as the suspension stroke which measures the relative motion between sprung and unsprung masses. Other significant measurements are sprung mass and unsprung accelerations. To develop an observer-based controller in this work, we only consider the suspension stroke as the measurable output, which can be easily measured by linear voltage differential transducers, potentiometers, etc., in practice.

\section{A. High Gain Observer}

The following HGO [27], [38] is proposed to estimate the states of the nonlinear suspension system (9)

$$
\dot{\hat{x}}=A \hat{x}+G(\hat{x}, U) U+\Phi(\hat{x}, t)+L\left(y-\hat{x}_{1}\right) .
$$

The observer gain matrix is designed as $L=\left[\begin{array}{lll}4 \mu & 6 \mu^{2} & 4 \mu^{3}\end{array}\right]$, with $\mu>0$ being the only tuning parameter for the observer. The scaled estimation error $e_{o}=\left[\begin{array}{lll}e_{o 1} & e_{o 2} & e_{o 3}\end{array}\right]$ can be defined as

$$
e_{o i}=\frac{1}{\mu^{(i-1)}}\left(x_{i}-\hat{x}_{i}\right)
$$


with $i=1,2,3$. To establish the convergence of the HGO, the following assumptions on the system dynamics need to be satisfied.

Assumption 1: The considered vehicle suspension system (9) is bounded-input bounded-state (BIBS) stable.

Assumption 2: The exogenous road input $f_{u}$ and its derivative are bounded.

Assumption 3: The maximum permissible input to the system is limited by a practical constrain

$$
U \leq|u|_{m}
$$

where $|u|_{m}$ is the maximum permissible value of the active force.

The vehicular suspension system operates under practical limits in the presence of active control $U$ to improve the system performance. Hence, it fulfills the BIBS stability in the operating region with its bounded states and bounded inputs. The road input is a practical entity that affects the vehicular system dynamics (9) and, hence, it is bounded. For a practical design, there exist limits on the active force output of the hydraulic actuator, which is a physical system. In such a scenario, the notion of bounded input with a maximum bound of $|u|_{m}$ has been considered. This assumption of bounded input is generally considered in the hydraulic [27], [41] and active suspension control [1], [8], [14] designs to ensure stable operations in practice.

Lemma 1: For the nonlinear suspension system (9) satisfying Assumptions 1-3, the hydraulic actuator nonlinearity is invertible for practical operating conditions.

Proof: The hydraulic actuator nonlinearity is given as

$$
g\left(x_{3}, U\right)=\lambda \sqrt{\frac{P_{s}-\operatorname{sign}(U) \frac{x_{3}}{M A_{p}}}{\rho} .}
$$

It is nonsingular except when $P_{s}=\frac{x_{3}}{M A_{p}} \operatorname{sign}(U)$ i.e., when the supply pressure is equivalent to the pressure difference across the actuator valves. However, for physical operating conditions of the hydraulic actuator, the pressure difference across valves does not exceed the supply pressure [34].

Theorem 1: For system (9) satisfying Assumptions 1-3 and employing observer (11), if for a positive parameter $\mu>0$, there exists a positive definite matrix $P$ such that

$$
(A-L C)^{T} P+P(A-L C)=-2 I
$$

then the estimation error $e_{o}$ is practically asymptotically stable since all error trajectories converge to an arbitrarily small neighborhood around the origin.

Proof: The estimation error dynamics from (9) and (11) can be written as

$$
\dot{e}_{o}=\left[\begin{array}{c}
\dot{e}_{o 1} \\
\dot{e}_{o 2} \\
\dot{e}_{o 3}
\end{array}\right]=\mu \tilde{A} e_{o}+G_{1} \frac{\tilde{\varphi_{a}}+f_{u}}{\mu}+G_{2} \frac{M \tilde{g} U+\tilde{\varphi_{b}}}{\mu^{2}}
$$

with

$$
\tilde{A}=\left[\begin{array}{lll}
-4 & 1 & 0 \\
-6 & 0 & 1 \\
-4 & 0 & 0
\end{array}\right], G_{1}=\left[\begin{array}{l}
0 \\
1 \\
0
\end{array}\right], G_{2}=\left[\begin{array}{l}
0 \\
0 \\
1
\end{array}\right] .
$$

The nonlinear terms are given as $\tilde{\varphi}_{a}=\varphi_{a}(x, t)-\varphi_{a}(\hat{x}, t), \tilde{\varphi}_{b}$ $=\varphi_{b}(x, t)-\varphi_{b}(\hat{x}, t)$, and $\tilde{g}=g(x, U)-g(\hat{x}, U)$. Consider the following Lyapunov function:

$$
V_{o}=\frac{1}{2} e_{o}^{T} P e_{o} .
$$

The time derivative of the Lyapunov function $V_{o}$ similar to [27], [38] can be written as

$$
\begin{aligned}
\dot{V}_{o}=-\mu\left\|e_{o}\right\|^{2}+\frac{1}{\mu} e_{o}^{T} P & G_{1} \tilde{\varphi}_{a}+\frac{1}{\mu} e_{o}^{T} P G_{1} f_{u} \\
& +\frac{1}{\mu^{2}} e_{o}^{T} P G_{2} \tilde{\varphi}_{b}+\frac{1}{\mu^{2}} e_{o}^{T} P G_{2} \tilde{g} U .
\end{aligned}
$$

For the nonlinear functions $\varphi_{a}$ and $\varphi_{b}$, the states $x_{1}, x_{2}$, and $x_{3}$ are BIBS stable within operating limits as per Assumption1 and, hence, bounded. The Lipschitz continuity of the nonlinear function $\varphi_{a}$ depends on $\varphi$. From (3), it can be seen that $\varphi$ is differentiable everywhere except at $x_{2}=0$. For all $x_{2} \neq 0$

$$
\left\{\begin{array}{l}
\left|\varphi_{a}\left(x_{1}, x_{2}\right)-\hat{\varphi}_{a}\left(\hat{x}_{1}, \hat{x}_{2}\right)\right| \leq l_{p 1}\left|e_{o 1}\right|+l_{p 2}\left|e_{o 2}\right| \\
\left|\varphi_{b}\left(x_{2}, x_{3}\right)-\hat{\varphi}_{b}\left(\hat{x}_{2}, \hat{x}_{3}\right)\right| \leq l_{p 3}\left|e_{o 2}\right|+l_{p 4}\left|e_{o 3}\right|
\end{array}\right.
$$

where $l_{p j}, j=1,2, \ldots, 4$ are the Lipschitz constants. Similarly, for the input nonlinearity $g\left(x_{3}, U\right)$ it can be seen from (13) that

$$
\mid g\left(x_{3}, U\right)-\hat{g}\left(\hat{x}_{3}, U\left|\leq l_{p 5}\right| e_{o 3} \mid\right.
$$

with the Lipschitz constant $l_{p 5}$. For details refer to the Appendix. Considering $L_{p}=\frac{1}{\mu^{2}}\left[\mu\left(l_{p 1}+l_{p 2}\right) \eta_{1}+\left(l_{p 3}+l_{p 4}\right) \eta_{2}\right]$ $+\frac{1}{\mu^{2}}\left[l_{p 5} \eta_{2}|u|_{m}\right]$, with $\eta_{k}=\left\|P G_{k}\right\|$ for $k=1,2$. Then we can write

$$
\dot{V}_{o} \leq-\left(\mu-L_{p}\right)\left\|e_{o}\right\|^{2}+\frac{1}{\mu} \eta_{1} f_{u}\left\|e_{o}\right\| .
$$

Consequently, we can, thus, write that the error $e_{o}$ converges to an ultimate bound as

$$
\left\|e_{o}\right\| \leq \frac{1}{\mu\left(\mu-L_{p}\right)} \eta_{1} f_{u} .
$$

A proper selection of $\mu$ such that the condition $\mu>L_{p}$ is satisfied ensures the ultimate boundedness (20) of the estimation error around the origin [27]. Further, by selecting a large $\mu$, the bound (20) can be effectively reduced. Although the estimation error convergence is asymptotic stable, the estimation error converges to the known bound (20) in finite-time.

Remark 1: For the nonlinear system (9), the solution is understood in Filippov sense due to the presence of discontinuity in the nonlinear functions $\varphi$ and $g\left(x_{3}, U\right)$. Consequently, the Lipschitz bounds for $\varphi_{a}$ can be computed locally as discussed earlier. Further, it can be analyzed that similar to the work in [20], the case $x_{2}=0$ appears locally or at the end due to the proposed HOSM controller in the following section.

Remark 2: For the designed HGO (11), with the estimation error dynamics (15), the solution of the Lyapunov equation (14) is dependent on the eigenvalues of the matrix $(A-L C)$. From the estimation error dynamics, the matrix $(A-L C)$ is given as

$$
A-L C=\left[\begin{array}{ccc}
-4 \mu & 1 & 0 \\
-6 \mu^{2} & 0 & 1 \\
-4 \mu^{3} & 0 & 0
\end{array}\right]
$$


For the design of the observer gain $\mu>0$, it can be ensured that the matrix $(A-L C)$ is Hurwitz with stable eigenvalues. In such a scenario, the solution of the Lyapunov equation (14) exists.

\section{B. Recursive Derivative Terminal Sliding Mode}

The estimated states of the suspension system (9) are now employed to design an output feedback controller based on the recursive derivative terminal sliding mode [33] approach. The design of the controller is initiated by considering the estimated tracking error for the suspension stroke $x_{1}$ as

$$
\epsilon_{0}=\hat{x}_{1}-x_{d}
$$

where $x_{d}$ is the desired reference trajectory. It can be deduced from the system dynamics (9), that the relative degree of the output error $\epsilon_{0}$ w.r.t. to the control input $U$ is 3. Consequently, we design the following recursive derivative terminal sliding mode [33] variables as

$$
\left\{\begin{array}{l}
\epsilon_{1}=\beta_{1} \epsilon_{0}+\dot{\epsilon}_{0}^{\gamma_{1}} \\
\epsilon_{2}=\beta_{2} \epsilon_{1}+\dot{\epsilon}_{1}^{\gamma_{2}}
\end{array}\right.
$$

with $\dot{\epsilon}_{1}=\gamma_{1} \dot{\epsilon}_{0}^{\left(\gamma_{1}-1\right)} \ddot{\epsilon}_{0}+\beta_{1} \dot{\epsilon}_{0}$. To obtain higher order derivatives of $\epsilon_{0}$, i.e., $\dot{\epsilon}_{0}$ and $\ddot{\epsilon}_{0}$, a second-order robust higher order sliding mode differentiator [39] is employed. The integral terminal sliding surface can be then designed as

$$
\sigma=\epsilon_{2}+\Gamma \int_{0}^{t} \epsilon_{2}^{\frac{1}{\gamma_{3}}} d t
$$

where $\gamma_{i}=\frac{p_{i}}{q_{i}}$ and $p_{i}>q_{i}>0$ are positive odd integers for $i=1,2,3$. The gains $\beta_{i}$ and $\Gamma$ are positive constants to be designed. The dynamics of the terminal sliding surface (23) is, thus, dependent on derivative of the error surface $\epsilon_{2}$. Furthermore, the dynamics of $\epsilon_{2}$ can be obtained by differentiating $\epsilon_{1}$, and that of $\epsilon_{1}$ by differentiating $\epsilon_{0}$. Hence, the design follows a recursive procedure, where successive derivatives are required. It can be deduced that $\epsilon_{1}$ needs to be differentiated twice while $\epsilon_{2}$ needs to be differentiated once for obtaining the sliding dynamics $\dot{\sigma}$. As the designed error surfaces $\epsilon_{1}$ and $\epsilon_{2}$ are nonlinear with exponential terms $\gamma_{1}$ and $\gamma_{2}$, it is mandatory to ensure that successive differentiations do not result in singularity. To ensure that there is no singularity problem when the controller is designed, the gains $\gamma_{i}$ are chosen such that the following condition is satisfied:

$$
\gamma_{i}>3-i
$$

where $i=1,2$. Employing this condition, it can be seen that $\gamma_{i}$ must be selected such that $\gamma_{1}>2$ and $\gamma_{2}>1$. Thus, even with successive differentiations of $\epsilon_{1}$ and $\epsilon_{2}$, singularity in the controller design would not arise. The control task is now to design a nonlinear robust output feedback control law which forces the sliding surface $\sigma$ to converge to the equilibrium point in finite time.

Theorem 2: For the suspension system (9) satisfying Assumptions 1-3, employing the estimated states using (11), we consider the following nonlinear control law

$$
U=\frac{1}{\Lambda M g\left(\hat{x}_{3}, U\right)}\left[-\Gamma \epsilon_{2}^{\frac{1}{\gamma_{3}}}-\Xi(\hat{x}, t)-\Psi_{x d}+\zeta(\sigma)\right]
$$

where $\Lambda, \Psi_{x d}$, and $\Xi(\hat{x, t})$ are discussed later. The robust control $\zeta(\sigma)$ given by

$$
\zeta(\sigma)=-\kappa_{1}|\sigma|^{\frac{1}{2}} \operatorname{sign}(\sigma)-\kappa_{2} \int_{0}^{t} \operatorname{sign}(\sigma) d t
$$

with positive gains $\kappa_{1}$ and $\kappa_{2}$ ensures the convergence of the sliding dynamics to a practically bounded region around the equilibrium point in finite time.

Proof: The dynamics of the sliding surface $\sigma$ can be written as

$$
\begin{aligned}
\dot{\sigma}= & \dot{\epsilon}_{2}+\Gamma \epsilon_{2}^{\frac{1}{\gamma_{3}}} \\
= & \Gamma \epsilon_{2}^{\frac{1}{\gamma_{3}}}+\beta_{2} \dot{\epsilon}_{1}+\gamma_{2} \dot{\epsilon}_{1}^{\left(\gamma_{2}-1\right)} \ddot{\epsilon}_{1} \\
= & \Gamma \epsilon_{2}^{\frac{1}{\gamma_{3}}}+\beta_{1} \beta_{2} \dot{\epsilon}_{0}+\left[\beta_{2} \gamma_{1} \dot{\epsilon}_{0}^{\left(\gamma_{1}-1\right)}+\beta_{1} \gamma_{2} \dot{\epsilon}_{1}^{\left(\gamma_{2}-1\right)}\right] \ddot{\epsilon}_{0} \\
& +\gamma_{2} \dot{\epsilon}_{1}^{\left(\gamma_{2}-1\right)}\left[\gamma_{1}\left(\gamma_{1}-1\right) \dot{\epsilon}_{0}^{\left(\gamma_{1}-2\right)}\left(\ddot{\epsilon}_{0}\right)^{2}+\gamma_{1} \dot{\epsilon}_{0}^{\left(\gamma_{1}-1\right)} \dddot{\epsilon}_{0}\right] \\
= & \Gamma \epsilon_{2}^{\frac{1}{\gamma_{3}}}+\beta_{1} \beta_{2} \dot{\epsilon}_{0}+\Psi_{1} \ddot{\epsilon}_{0}+\Psi_{2}\left(\ddot{\epsilon}_{0}\right)^{2}+\Psi_{3} \dddot{\epsilon}_{0}
\end{aligned}
$$

with $\Psi_{1}=\beta_{2} \gamma_{1} \dot{\epsilon}_{0}^{\left(\gamma_{1}-1\right)}+\beta_{1} \gamma_{2} \dot{\epsilon}_{1}^{\left(\gamma_{2}-1\right)}, \quad \Psi_{2}=\gamma_{2} \gamma_{1}\left(\gamma_{1}-1\right)$ $\dot{\epsilon}_{1}^{\left(\gamma_{2}-1\right)} \dot{\epsilon}_{0}^{\left(\gamma_{1}-2\right)}$, and $\Psi_{3}=\gamma_{1} \gamma_{2} \dot{\epsilon}_{1}^{\left(\gamma_{2}-1\right)} \dot{\epsilon}_{0}^{\left(\gamma_{1}-1\right)}$. From the observer dynamics (11) and (21), the following can be obtained:

$$
\left\{\begin{array}{l}
\dot{\epsilon}_{0}=\hat{x}_{2}-\dot{x}_{d}+\delta_{1} \\
\ddot{\epsilon}_{0}=\hat{x}_{3}+\varphi_{a}(\hat{x}, t)-\ddot{x}_{d}+\delta_{2} \\
\dddot{\epsilon}_{0}=\varphi_{b}(\hat{x}, t)+M g\left(\hat{x}_{3}, U\right) U-\dddot{x}_{d}+\delta_{3}
\end{array}\right.
$$

where the perturbation terms $\delta_{i}$ are given as

$$
\left\{\begin{aligned}
\delta_{1}= & 4 \mu e_{o 1} \\
\delta_{2}= & 4 \mu^{2} e_{o 2}-10 \mu^{2} e_{o 1} \\
\delta_{3}= & 4 \mu^{3} e_{o 3}-10 \mu^{3} e_{o 2}+16 \mu^{3} e_{o 1}+\dot{\varphi}_{a}(\hat{x}, t) \\
& +4 \mu \tilde{\varphi}_{a}+4 \mu f_{u}+4 \mu \dot{f}_{u} .
\end{aligned}\right.
$$

Substituting for $\dot{\epsilon}_{0}, \ddot{\epsilon}_{0}$, and $\dddot{\epsilon}_{0}$, the sliding dynamics can be expressed as

$$
\begin{gathered}
\dot{\sigma}=\Gamma \epsilon_{2}^{\frac{1}{\gamma_{3}}}+\Xi(\hat{x}, t)+\Psi_{3} M g\left(\hat{x}_{3}, U\right) U+\beta_{1} \beta_{2} \delta_{+} \Psi_{1} \delta_{2} \\
+\Psi_{2} f\left(\delta_{2}, \hat{x}\right)+\Psi_{3} \delta_{3}
\end{gathered}
$$

with $\Xi(\hat{x}, t)=\beta_{1} \beta_{2} f_{1}\left(\hat{x}_{2}, t\right)+\Psi_{1} f_{2}(\hat{x}, t)+\Psi_{2} f_{3}(\hat{x}, t)+$ $\Psi_{3} f_{4}(\hat{x}, t), \quad \Psi_{x d}=-\beta_{1} \beta_{2} \dot{x}_{d}-\Psi_{1} \ddot{x}_{d}-\Psi_{3} \dddot{x}_{d}, \quad f_{1}\left(\hat{x}_{2}, t\right)=$ $\hat{x}_{2}, \quad f_{2}(\hat{x}, t)=\hat{x}_{3}+\varphi_{a}(\hat{x}, t), \quad f_{3}=\left[\hat{x}_{3}+\varphi_{a}(\hat{x}, t)-\ddot{x}_{d}\right]^{2}$, and $f_{4}(\hat{x}, t)=\varphi_{b}(\hat{x}, t)$ and with $f\left(\delta_{2}, \hat{x}\right)$ being a nonlinear function of the estimated states and the perturbation $\delta_{2}$. In the above-mentioned expression, the sliding dynamics are a function of the error surface $\epsilon_{2}$, the estimated state term $\Xi(\hat{x}, t)$, the nonlinearities $g\left(\hat{x}_{3}, U\right)$ and $\Psi_{3}$, the control input $U$, the reference trajectory, and the terms which are functions of the perturbations $\delta_{1}, \delta_{2}$, and $\delta_{3}$. The terms which are functions of $\delta_{1}, \delta_{2}$, and $\delta_{3}$ can be then lumped together in terms of a single 
perturbation as

$$
\Delta_{c}=\beta_{1} \beta_{2} \delta_{1}+\Psi_{1} \delta_{2}+\Psi_{2} f\left(\delta_{2}, \hat{x}\right)+\Psi_{3} \delta_{3} .
$$

The sliding dynamics can be, hence, written as

$$
\dot{\sigma}=\Gamma \epsilon_{2}^{\frac{1}{\gamma_{3}}}+\Xi(\hat{x}, t)+\Psi_{3} M g\left(\hat{x}_{3}, U\right) U+\Psi_{\mathrm{xd}}+\Delta_{c} .
$$

From Lemma 1, it can be established that $g\left(\hat{x}_{3}, U\right)$ is invertible. Let us consider similar to [33]

$$
\Omega \equiv\left\{\begin{array}{lc}
\prod_{j=1}^{2} \dot{\epsilon}_{j-1}^{\frac{p_{j}}{q_{j}}-1}, & \text { for } \\
\nu, & \text { otherwise }
\end{array}\left|\prod_{j=1}^{2} \dot{\epsilon}_{j-1}^{\frac{p_{j}}{q_{j}}-1}\right| \geq \nu\right.
$$

with $\nu>0$. For the control law (25), we set

$$
\Lambda=\gamma_{1} \gamma_{2} \Omega
$$

Condition (32) can be seen as a saturated function for $\Psi_{3}$ and results in two scenarios. In the first case when $\left|\prod_{j=1}^{2} \dot{\epsilon}_{j-1}^{\frac{p_{j}}{q_{j}}-1}\right| \geq$ $\nu$, the nonlinear function $\Psi_{3}$ is not approximated and directly employed for $\Lambda$. Therefore, applying the control law (25), the sliding dynamics can be expressed as

$$
\dot{\sigma}=\zeta(\sigma)+\Delta_{c}
$$

when $\left|\prod_{j=1}^{2} \dot{\epsilon}_{j-1}^{\frac{p_{j}}{q_{j}}-1}\right| \geq \nu$. In this scenario, to design the gains $\kappa_{1}$ and $\kappa_{2}$ which ensure the practical convergence of the sliding surface, the bound of $\Delta_{c}$ is required. It can be seen from (30) that the perturbation $\Delta_{c}$ is a function of the estimated states of the system and the perturbations $\delta_{1}, \delta_{2}$, and $\delta_{3}$. The perturbations $\delta_{1}$ and $\delta_{2}$ are the functions of the observation errors $e_{01}$ and $e_{o 2}$ as established in (28). Since the estimated states of the system are bounded by practical constraints, the derivative of $\delta_{1}$ and $\delta_{2}$ is bounded. Similarly, the nonlinear function $f\left(\delta_{2}, \hat{x}\right)$ is a function of the bounded estimated states and the bounded perturbation $\delta_{2}$. The perturbation $\delta_{3}$ is a function of estimation errors, the exogenous road input $f_{u}$, and the estimated state dynamics. Under boundedness assumptions of the estimated state dynamics and the road input as discussed in Assumptions 1 and 2, the bound of the time derivative of the perturbation can be established. Hence, we can establish, that bound of the time derivative of the perturbation $\Delta_{c}$ can be given as

$$
\left|\dot{\Delta}_{c}\right| \leq \rho_{d}
$$

where $\rho_{d}>0$ is the cumulative bound of the all the components of $\Delta_{c}$ as discussed earlier. Now, consider the following Lyapunov function:

$$
V_{c}=\Sigma^{T} P_{c} \Sigma
$$

where $\Sigma^{T}=\left[|\sigma|^{\frac{1}{2}} \operatorname{sign}(\sigma) \dot{\sigma}\right]$ and $P_{c}$ is a positive definite matrix defined as

$$
P_{c}=\frac{\kappa_{1}}{2}\left[\begin{array}{cc}
4 \kappa_{2}+\kappa_{1}^{2} & -\kappa_{1} \\
-\kappa_{1} & 2
\end{array}\right]
$$

The time derivative of the Lyapunov function can then be computed similar to [31] and [32] as

$$
\dot{V}_{c} \leq-\frac{1}{|\sigma|^{\frac{1}{2}}} \Sigma^{T} Q \Sigma
$$

where $Q$ is a positive definite matrix given as

$$
Q=\frac{\kappa_{1}}{2}\left[\begin{array}{cc}
2 \kappa_{2}+\kappa_{1}^{2}-2 \rho_{d} & \star \\
-\left(k_{1}+\frac{2 \rho_{d}}{\kappa_{1}}\right) & 1
\end{array}\right] .
$$

To ensure that matrix $Q$ is positive definite, the gains $\kappa_{1}$ and $\kappa_{2}$ are selected as

$$
\kappa_{1}>0, \kappa_{2}>\frac{6 \rho_{d}+4\left(\rho_{d} / \kappa_{1}\right)^{2}}{2} .
$$

Employing the fact that $|\sigma|^{\frac{1}{2}} \leq\|\Sigma\|_{2}, \dot{V}_{c}$ can be written as [31], [32]

$$
\dot{V}_{c} \leq-\lambda_{\min }(Q)\|\Sigma\|
$$

It can, thus, be established that the sliding surface converges to a bound $|\sigma| \leq \xi_{1}$ while the dynamics converge to a bound $|\dot{\sigma}| \leq \bar{\nu}_{1}$. The computation of these bounds are dependent on the magnitude of the saturation constant $\nu$ discussed in (32). In the second scenario when $\left|\prod_{j=1}^{2} \dot{\epsilon}_{j-1}^{\frac{p_{j}}{q_{j}}-1}\right|<\nu$, the saturated value of $\Psi_{3}$, i.e., $\nu$ is considered. In such a scenario, the sliding dynamics can be approximated as

$$
|\dot{\sigma}| \leq f\left(\hat{x}, \rho_{d}, \nu\right)=\bar{\nu}_{2} .
$$

The sliding variable $\sigma$ enters the bound $|\sigma| \leq \xi_{2}$ in a finite time. However, with the evolution of the dynamics of the sliding surface, the sliding variable ultimately leaves this bound. It can always be guaranteed that the sliding variable then stays in the larger domain of $|\sigma| \leq \xi_{1}$, where $\xi_{1}>\xi_{2}$ resulting in a practical sliding mode. Thus, integrating both scenarios, we can express that

$$
|\dot{\sigma}| \leq \max \left(\bar{\nu}_{1}, \overline{\nu_{2}}\right)=\nu_{2}
$$

Hence, the convergence of the sliding dynamics to the ultimate bound in finite-time is established.

\section{Closed-Loop Stability Analysis}

As the ultimate boundedness of the observer in (20) and the sliding dynamics in (37) are established in finite-time, the practical stability of the combined observer and controller dynamics is established based on these bounds. Similar to [42]-[44], the closed-loop stability is established in two phases as follows.

1) In the first phase, based on initial conditions of the observer and states, the estimation error converges to an ultimate finite bound as shown in (20) in Theorem 1. During this phase, under BIBS assumptions, Assumption 1 and boundedness of the road disturbances, it can be shown easily that the closed-loop system states remain bounded.

2) After the observer has converged to the ultimate bound in finite-time, one can then establish the practical boundedness of the sliding surface $\sigma$, with the estimated states as discussed in Theorem 2 . 
3) In the second phase, after the observer and sliding surfaces converge to their respective ultimate bounds in finite-time sequentially, the practical stability of the combined observer-controller dynamics is then established.

To establish the closed-loop stability, consider the following Lyapunov candidate function:

$$
\begin{aligned}
V & =\frac{1}{2} e_{o}^{T} P e_{o}+\Sigma^{T} P_{c} \Sigma \\
& =V_{o}+V_{c} .
\end{aligned}
$$

Considering the case when $\left|\prod_{j=1}^{2} \frac{\frac{p}{j}_{\epsilon_{j}}}{q_{j-1}}\right| \geq \nu$, from (19) and (37) $\dot{V}$ can, thus, be written as

$$
\dot{V} \leq-\left(\mu-L_{p}\right)\left\|e_{o}\right\|^{2}-\lambda_{\min }(Q)\|\Sigma\|+\frac{1}{\mu} \eta_{1} f_{u}\left\|e_{o}\right\| .
$$

Now, consider the new vector $Z=\left[\left\|e_{o}\right\|\|\Sigma\|^{\frac{1}{2}}\right]$ and define $a=\left(\mu-L_{p}\right)$ and $b=\lambda_{\min }(Q), \dot{V}$ can be written as

$$
\begin{array}{r}
\dot{V} \leq-\left(a_{1}\left\|e_{o}\right\|-b_{1}\|\Sigma\|^{\frac{1}{2}}\right)^{2}-\left(a-a_{1}^{2}\right)\left\|e_{o}\right\|^{2}-\left(b-b_{1}^{2}\right)|\Sigma| \\
+\frac{1}{\mu} \eta_{1} f_{u}\left\|e_{o}\right\|-2 a_{1} b_{1}\left\|e_{o}\right\||\Sigma|^{\frac{1}{2}}
\end{array}
$$

with $a_{1} b_{1}=\frac{1}{2}$. Now selecting $a=\left(\kappa+a_{1}^{2}\right)$ and $b=\left(\kappa+b_{1}^{2}\right)$, $\dot{V}$ can be written as

$$
\begin{aligned}
\dot{V} \leq & -\left(a_{1}\left\|e_{o}\right\|-b_{1}\|\Sigma\|^{\frac{1}{2}}\right)^{2}-\kappa\left[\left\|e_{o}\right\|^{2}+\|\Sigma\|^{\frac{1}{2}}\right] \\
& +\frac{1}{\mu} \eta_{1} f_{u}\left\|e_{o}\right\|-2 a_{1} b_{1}\left\|e_{o}\right\| \Sigma \|^{\frac{1}{2}} \\
\leq & -\kappa\left[\left\|e_{o}\right\|^{2}+\|\Sigma\|^{\frac{1}{2}}\right]+\frac{1}{\mu} \eta_{1} f_{u}\left\|e_{o}\right\| \\
& -2 a_{1} b_{1}\left\|e_{o}\right\| \Sigma \|^{\frac{1}{2}} \\
\leq & -\kappa\|Z\|^{2}+\kappa_{p}\|Z\|
\end{aligned}
$$

where $\kappa>0$ is a positive constant. To show that the term $-\kappa\|Z\|^{2}$ dominates the function $\dot{V}$, we can write for $0<\theta<1$

$$
\dot{V} \leq-\kappa(1-\theta)\|Z\|^{2}-\kappa\|Z\|^{2}+\kappa_{p}\|Z\| .
$$

Consequently, for $\|Z\| \geq \frac{\kappa_{p}}{\kappa \theta}$, it can be shown that

$$
\dot{V} \leq-\kappa(1-\theta)\|Z\|^{2} .
$$

Thus, the radius to which the closed-loop system converges can now be obtained as $\alpha=\sqrt{\frac{\kappa_{p}}{\kappa \theta}}$. For a large value of the observer gain $\mu$, the radius of convergence $\alpha$ can be effectively reduced by tuning the parameters $\nu, \kappa_{1}$, and $\kappa_{2}$. Hence, local practical stability [19], [20] of the closed-loop system holds.

Remark 3: With the convergence of $\sigma$ to a practical bound, it can be established that $\epsilon_{0}$ is also practically stable using the derivative terminal sliding mode. Further, for the controlled plant $\left(x_{1}-x_{d}\right)$ is also practically stable.

\section{Simulation Results and Discussion}

To evaluate the effectiveness of the proposed output feedback controller, the quarter vehicle parameters of a Class D Sedan were considered and simulation was performed.
TABLE I

QUARTER VEHICLE AND HydRAULIC ACTUATOR PARAmeters

\begin{tabular}{lcc}
\hline \hline & Parameter & Value \\
\hline & Sprung mass $\left(m_{s}\right)$ & $342.5 \mathrm{~kg}$ \\
Quarter & Unsprung mass $\left(m_{u}\right)$ & $40 \mathrm{~kg}$ \\
Vehicle & Suspension spring stiffness $\left(k_{s}\right)$ & $153000 \mathrm{~N} / \mathrm{m}$ \\
& Suspension damper $\left(b_{s}\right)$ & $1000 \mathrm{~N} \& \mathrm{middot} ; \mathrm{s} / \mathrm{m}$ \\
& Tire damping $\left(k_{t}\right)$ & $14.6 \mathrm{~N} / \mathrm{m}$ \\
& Tire stiffness $\left(b_{t}\right)$ & $268000 \mathrm{~N} / \mathrm{m}$ \\
Hydraulic & Supply pressure $\left(P_{s}\right)$ & $1.034 \times 10^{7} \mathrm{~N} / \mathrm{m}^{2}$ \\
Actuator [34] & Piston area $\left(A_{p}\right)$ & $3.35 \times 10^{-4}$ \\
& Discharge coefficient $\left(C_{d}\right)$ & 0.61 \\
& Density of oil $(\rho)$ & $858 \mathrm{~kg} / \mathrm{m}^{3}$ \\
& $w$ & $1.436 \times 10^{-2} \mathrm{~m}$ \\
& $\alpha$ & $1.9143 \times 10^{13}$ \\
\hline \hline
\end{tabular}

\section{A. Parameter Selection}

The quarter vehicle suspension system with the vehicular and hydraulic actuator parameters given in Table I was considered in this work.

The initial conditions of the plant (9) and the designed HGO (11) were chosen as $x=\left[\begin{array}{lll}0 & 0 & 0\end{array}\right]^{T}$ and $\hat{x}=$ $\left[\begin{array}{lll}0.001 & 0 & 0.0001\end{array}\right]^{T}$, respectively. The nonlinearity in the suspension, spring, and stiffness coefficients are considered as $10 \%$ of their linear values. Thus, for simulation purposes $k_{n}=0.1 k_{s}$ and $b_{n}=0.1 b_{s}$ were selected. For the HGO, the gain bandwidth was selected as $\mu=380$. The selected $\mu$ is high enough to ensure the practical asymptotic stability of the observer. In the suspension system analysis, there has been no established reference trajectory employed as all measurements are relative. For the controller design, we consider the reference to be $x_{d}=0$. Since the established controller converges to a bound, hence the controlled suspension stroke is never zero and is within the design requirements. For the design of the recursive derivation subsystems (22), the gains were selected as $\beta_{1}=0.1, \beta_{2}=1$, $\gamma_{1}=0.33$, and $\gamma_{2}=1.66$, with $p_{1}=7, p_{2}=5, q_{1}=3$, and $q_{2}=3$. It can be seen that these parameters obey (24), thus ensuring that there is no singularity in the design of the control. For the design of the NSTSM sliding surface, $\Gamma=0.01, p_{3}=3$, and $q_{3}=1$ were chosen. The gains of the robust HOSM controller (26) were selected as $\kappa_{1}=50$ and $\kappa_{2}=500$.

\section{B. Simulations: Road Conditions, Uncertainties, and Sensor noise}

Simulations were carried out for various road conditions, parametric uncertainties, and sensor noise. For illustration purposes, the results for the vehicle traveling at a speed of $72 \mathrm{~km} / \mathrm{h}$, i.e., $v_{x}=20 \mathrm{~m} / \mathrm{s}$ under poor road (Class C, $\sigma_{r}=$ $256 \times 10^{-6} \mathrm{~m}^{3}$ ) [35] have been presented. To simulate the effect of road defect, we consider the following input [2]:

$$
z_{\text {def }}=\left\{\begin{array}{cl}
\frac{h[1-\cos 8 \pi t]}{2}, & 1 \leq t \leq 1.25 \\
0, & \text { otherwise }
\end{array}\right.
$$

where the height of the road bump is given as $h=4 \mathrm{~cm}$. Under such road and driving conditions, the performance of the state estimation employing the HGO (11) is presented in Fig. 1. 


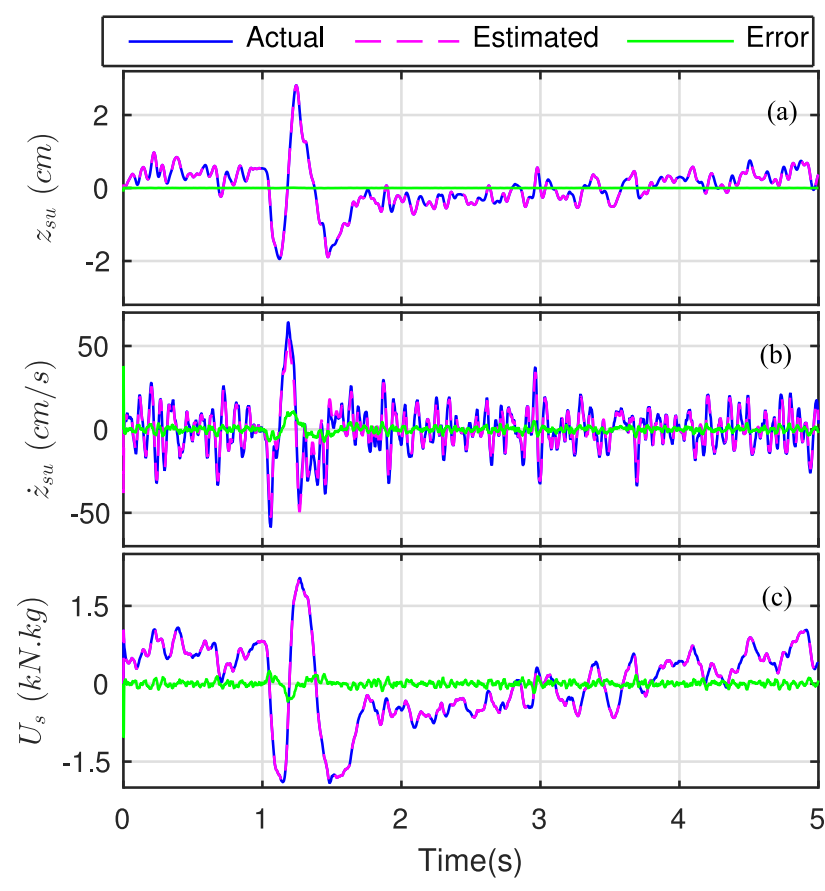

Fig. 1. Estimated states using the $\mathrm{HGO}$ (a) $z_{\mathrm{su}}$ and (b) $\dot{z}_{\mathrm{su}}$ (c) $M U_{s}$.

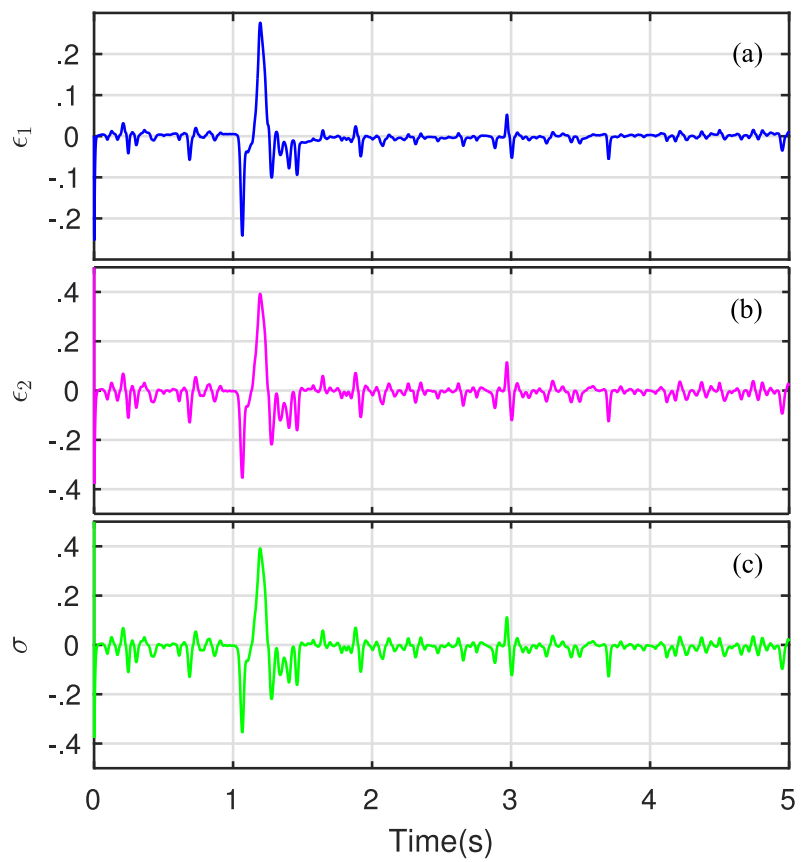

Fig. 2. Sliding surfaces (a) $\epsilon_{1}$, (b) $\epsilon_{2}$, and (c) $\sigma$.

In Fig. 1, along with the estimated states, the corresponding errors are also plotted to show the effectiveness of the proposed HGO in estimating the states in the presence of nonlinearities. It can be seen that all the estimation errors converge to a finite bound around the origin, leading to a practical stability. Employing the estimated states, the proposed NSTSM controller was designed and the convergence of the sliding states to a finite bound is shown in Fig. 2. Further, the closed-loop stability of the system is shown in Fig. 3. For the considered simulation conditions, the Lipschitz constants for the nonlinear functions

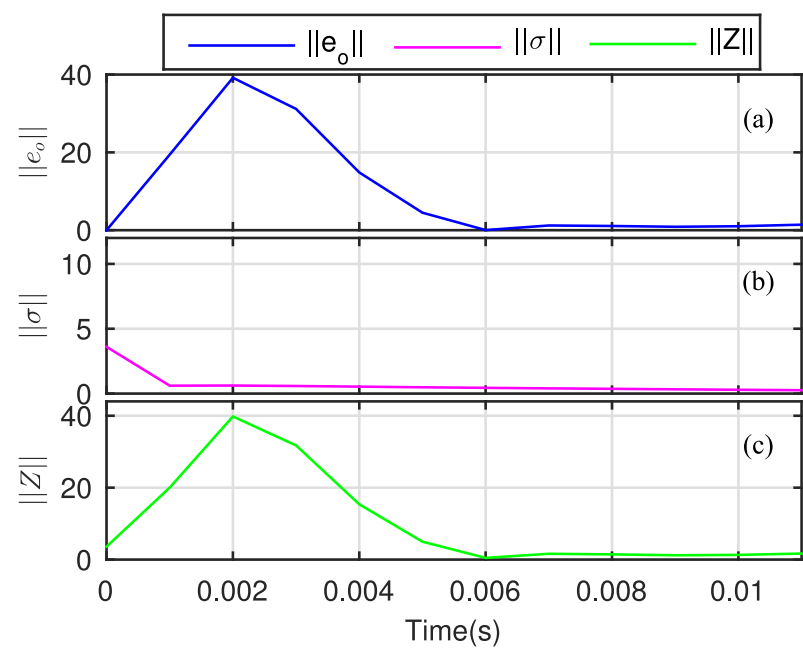

Fig. 3. Norm of (a) estimation error $\left\|e_{o}\right\|$, (b) sliding surface $\|\sigma\|$, and (c) closed-loop system $\|Z\|$.

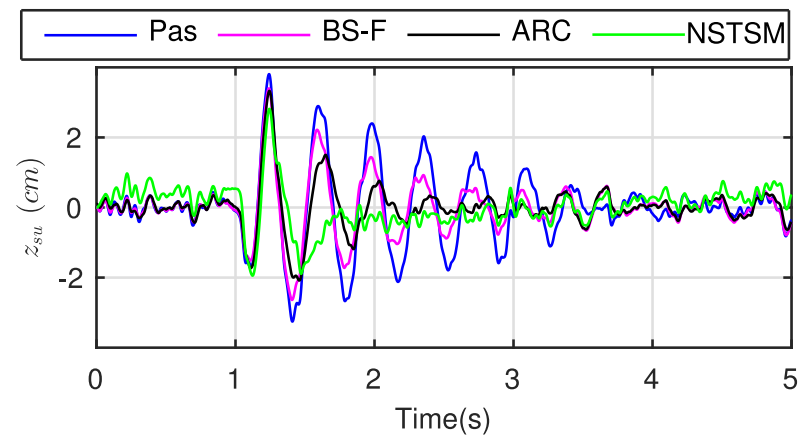

Fig. 4. Suspension stroke $z_{\text {su }}$ for the passive system, active backstepping feedback [27], controlled ARC [40], and controlled NSTSM.

were computed and the parameter $L_{p}$ (19) was computed as 141.9. Consequently, the ultimate bound of the estimation error (20) was computed as 4.9261. It can be seen that the estimation error converges to this bound in a finite time of $0.006 \mathrm{~s}$, as shown in Fig. 3(a). During this phase, under BIBS assumptions the states are bounded and the sliding surface converges in finite-time, as shown in Fig. 3(b). Consequently, after the estimated states converge to the finite bound, the closed-loop system also converges to the finite bound, as shown in Fig. 3(c). Thus, the closed-loop system's practical stability is established as discussed earlier.

With the convergence of the sliding surface established, the performance of the proposed controller was then compared to the passive suspension, backstepping approach based on the feedback gains (BS-F) active controlled suspension system and adaptive robust controlled (ARC) active suspension system. The improvement in suspension stroke, sprung mass acceleration, and tire deflection due to the applied NSTSM control is shown in Figs. 4-6.

It can be deduced from Figs. 5 and 6 that with the provided output feedback control there is a significant improvement in the passenger comfort and road holding as indicated by the reduction in the sprung mass acceleration and tire deflection, respectively. Employing the measure (8), the performance of 


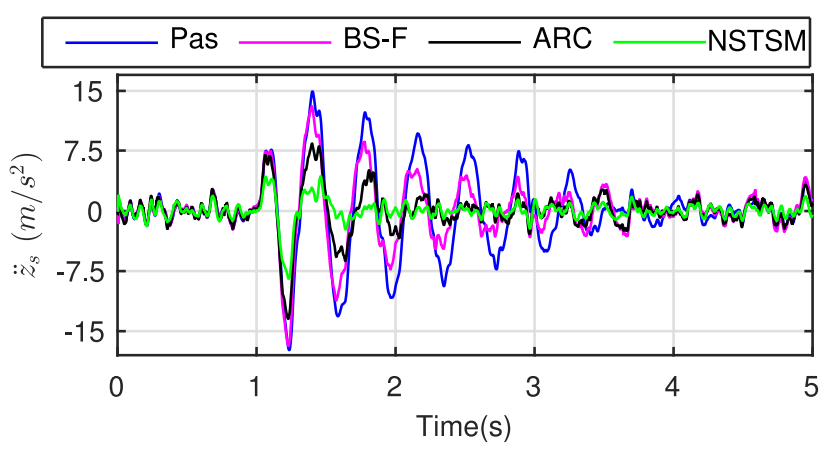

Fig. 5. Sprung mass acceleration $\ddot{z}_{s}$ for the passive system, active backstepping feedback [27], controlled ARC [40], and controlled NSTSM.

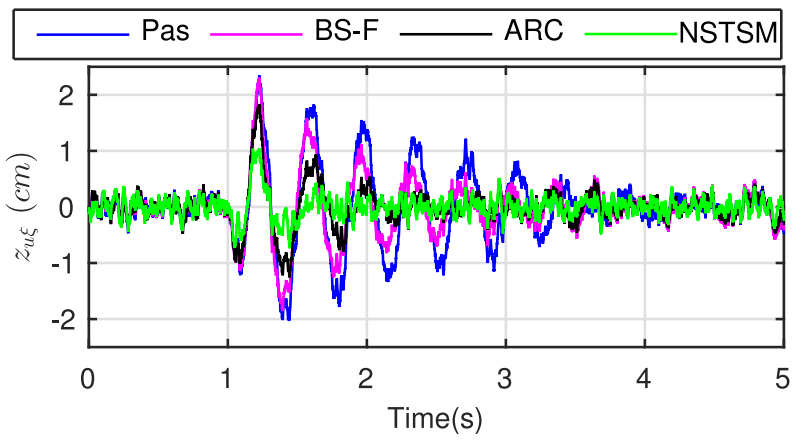

Fig. 6. Tire deflection $z_{u \xi}$ for the passive system, active backstepping feedback [27], controlled ARC [40], and controlled NSTSM.

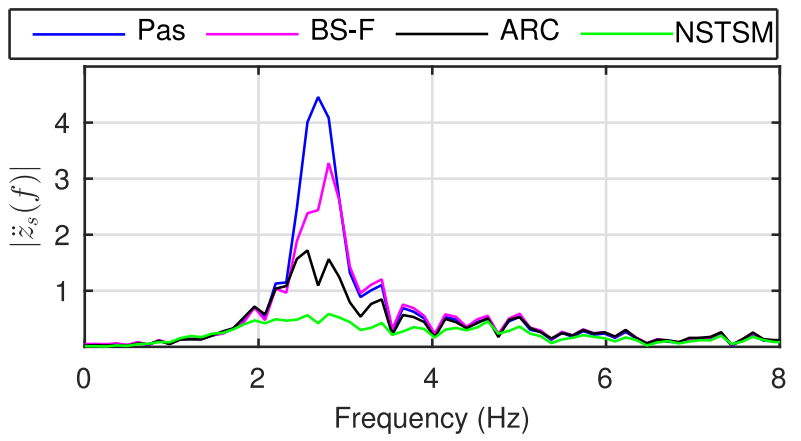

Fig. 7. FFT of sprung mass acceleration $\ddot{z}_{s}$ for the passive system, active backstepping feedback [27], controlled ARC [40], and controlled NSTSM.

the active suspension was improved by $73.59 \%$ in regards with passenger comfort and by $69.37 \%$ in regards with road holding. The improvement in the suspension performance in the desired range of frequency, i.e., $0-8 \mathrm{~Hz}$ is shown in Figs. 7 and 8 . It can be seen from Figs. 4-8 that the proposed NSTSM-HOSM controller in this paper outperforms the backstepping-feedback gains control approach and the adaptive robust control approach in both time and frequency domains. To evaluate the performance under the effect of uncertainties, the suspension system parameters, i.e., sprung mass, spring stiffness, and damping coefficient were considered to have variable values under the effect of random uncertainties. Sensor noise was added to the mea-sured suspension stroke and performance was evaluated. The performance measure for suspension deflection $\left(P_{z_{\text {sul }}}\right)$, sprung

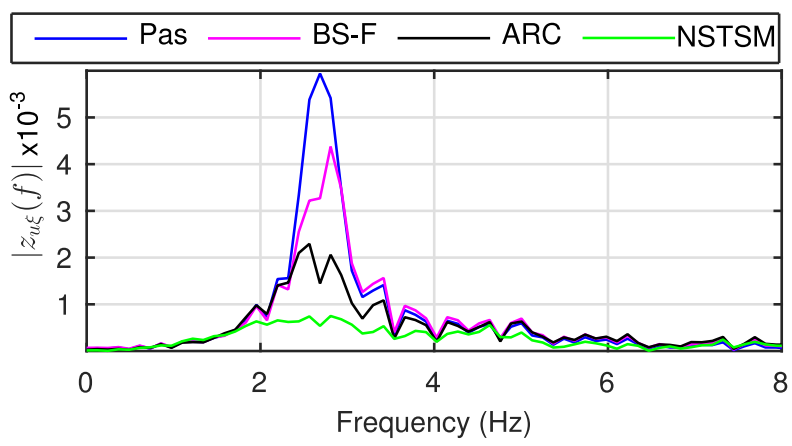

Fig. 8. FFT of tire deflection $z_{u \xi}$ for the passive system, active backstepping feedback [27], controlled ARC [40], and controlled NSTSM.

TABLE II

Performance Evaluation OVer Various Conditions

\begin{tabular}{lcccc}
\hline \hline Case & Conditions & $P_{\ddot{z}_{s}}$ & $P_{z_{\mathrm{su}}}$ & $P_{z_{u \xi}}$ \\
\hline Parametric & $5 \%$ & 0.7051 & 0.2996 & 0.6828 \\
Uncertainty & $7.5 \%$ & 0.6817 & 0.3199 & 0.6667 \\
Sensor & $1.1 \times 10^{-5}$ & 0.7230 & 0.3596 & 0.6876 \\
Noise & $3.3 \times 10^{-5}$ & 0.7005 & 0.4454 & 0.6729 \\
Varying & $60 \mathrm{~km} / \mathrm{h}$ & 0.7405 & 0.4201 & 0.7040 \\
Velocity & $100 \mathrm{~km} / \mathrm{h}$ & 0.7244 & 0.4186 & 0.6721 \\
(Class C-Road) & $150 \mathrm{~km} / \mathrm{h}$ & 0.7057 & 0.3840 & 0.6405 \\
Road & Class A & 0.7466 & 0.2790 & 0.7530 \\
Class & Class B & 0.7467 & 0.3441 & 0.7427 \\
$\left(v_{x}=20 \mathrm{~m} / \mathrm{s}\right)$ & Class C & 0.7359 & 0.4237 & 0.6937 \\
Class D & 0.6845 & 0.4418 & 0.5673 & \\
\hline
\end{tabular}

mass acceleration $\left(P_{\ddot{z}_{s}}\right)$, and tire deflection $\left(P_{z_{u \xi}}\right)$ for each of the above scenarios is presented in Table II.

It can be deduced from Table II that the proposed output feedback controller provides efficient active suspension control performance under various road as well as driving conditions.

\section{Discussion}

The implementation of the proposed output feedback control to improve passenger comfort on a practical suspension has to comply with several issues such as road holding, sensor noise, robustness, observer-controller stability, etc. The performance analysis of the proposed controller has indicated improvement in both passenger comfort and road holding as deduced from Table II. The RMS value of the dynamic wheel load was obtained as $616.4 \mathrm{~N}$, which is less than one third of the static load $1071.6 \mathrm{~N}$, thus satisfying the road holding criterion (7). Further, from Fig. 1(a) it can be seen that the provided control action maintains the suspension deflection below the required limit of $\pm 0.05 \mathrm{~m}$. The bounds on the maximum permissible control input can be computed as $550 \mathrm{~N}$ with a peak value of $2200 \mathrm{~N}$, which is a practical value similar to the work discussed in [34]. With regards to the sensor noise and parametric uncertainty, the robustness of the proposed controller is shown in Table II. In this work, we consider the HGO to estimate the states of the system. The significant aspects of the observer gain selection can be decided on the basis of (1) the peaking phenomena and (2) estimation, when there is sudden disturbance due to road defects. The choice of the observer gain affects the estimation performance, which ultimately affects the controller design. In 

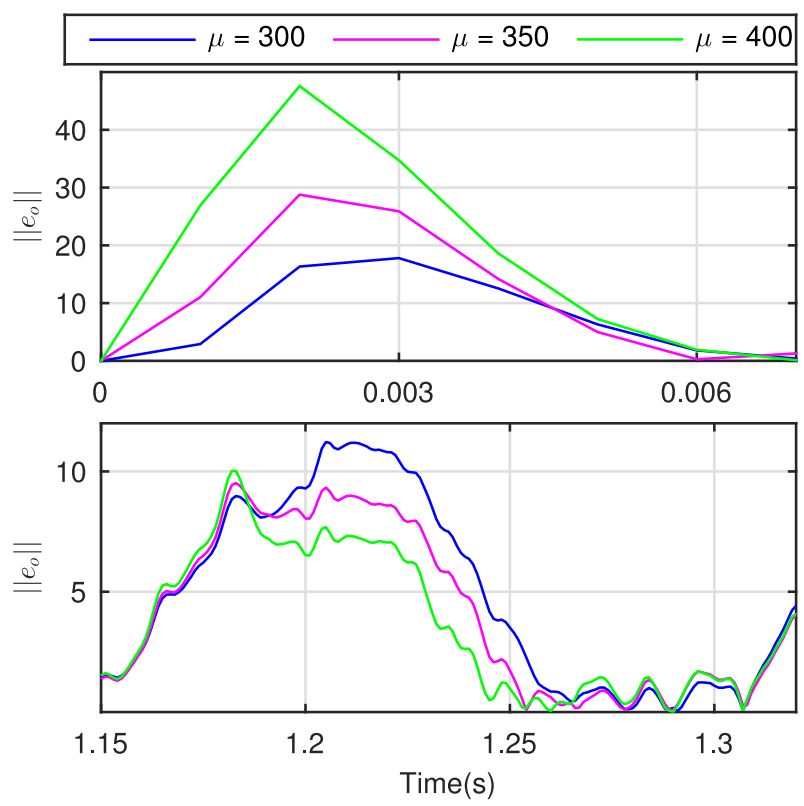

Fig. 9. Effect of different $\mu$ (a) peaking phenomena and (b) when road defects appear.

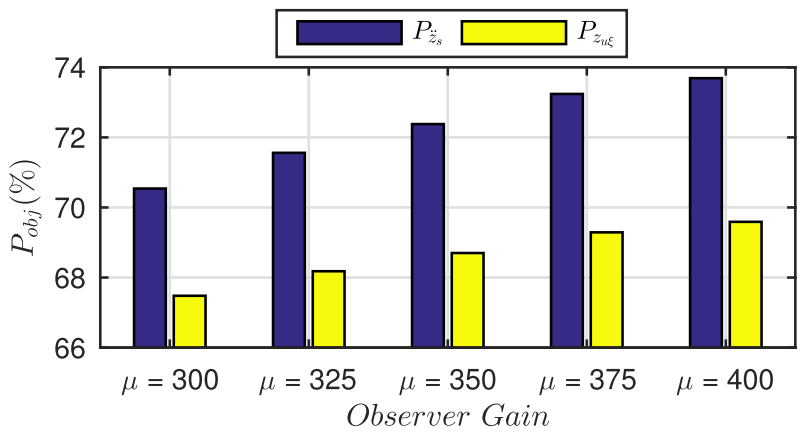

Fig. 10. Variation in performance indexes $P_{\ddot{z}_{s}}$ and $P_{z_{u \xi}}$ to with respect the observer gain $\mu$.

Fig. 9(a), the estimation error norm $\left\|e_{o}\right\|$ for different values of the observer gain $\mu$ is shown. It can be seen that by increasing the observer gain $\mu$ the peaking initial estimation error norm increases, i.e., the peaking effect is more pronounced. However, if we consider the estimation error when sudden disturbance, i.e., road inputs appear then it can be seen that the selection of a high $\mu$ is more effective as shown in Fig. 9(b).

With the increase of the observer gain, the performance of the closed-loop observer-controller system increases. This performance enhancement is reflected in the passenger comfort $\left(P_{\ddot{z}_{s}}\right)$ and road holding $\left(P_{z_{u}}\right)$ indexes as seen in Fig. 10. However, after value of $\mu=380$, there is no significant improvement in the performance of the controlled system. Although a high value of the observer gain $\mu$ provides better performance, it also results in peaking phenomena that adversely affect the controller performance. Further, in the presence of the sensor noise, a very high value of $\mu$ would result in high chattering for the sliding surface. Thus, the observer gain has to be tuned such that the control performance is maintained while ensuring that adverse effects of the peaking phenomena, chattering in sliding surface, and sensitivity to sensor noise are accounted and minimized.

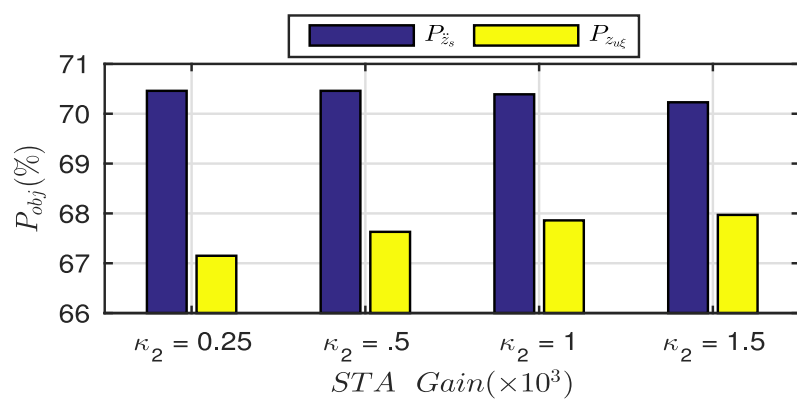

Fig. 11. Variation in performance indexes $P_{\ddot{z}_{s}}$ and $P_{z_{u \xi}}$ to with respect the STA gain $\kappa_{2}$.

We consider the suspension stroke sensor as the only output while designing the HGO in this work. Thus, the effect of the sensor noise on the HGO is greatly reduced in comparison to approaches where more noisy sensors such as accelerometers are employed. The implementation of the HGO-type observer in [27] for a hydraulic actuator further supplements the real-time applicability of this observer to the suspension control problem. The uncertainty in parameters discussed in this paper are related to mass and suspension parameter variations that arise due to various operating scenarios. These variations can be reflected in real-time suspension control as the effect of variable mass loading, effect of shocks, nonlinearity in spring, etc.

The proposed observer-based output feedback control approach in this paper also stresses on the aspects of ease of design and practical convergence. Similar to the backstepping, which is widely accepted as one of the most feasible approaches for active suspension control, a recursive derivative terminal sliding mode technique was discussed in this work. The influence of the controller gain $\kappa_{2}$ on the performance indexes $\left(P_{\ddot{z}_{s}}\right)$ and $\left(P_{z_{u}}\right)$ is shown in Fig. 11.

It can be seen that with the increase of $\kappa_{2}$, there is a minimal change in the passenger comfort as reflected from $P_{\ddot{z}_{s}}$. However, the road holding capability, i.e., $P_{z_{u \xi}}$ is better when $\kappa_{2}$ is larger. This is in accordance with the suspension system, where both the objectives of the passenger comfort and road holding cannot be simultaneously enhanced. The influence of the gain $\kappa_{1}$ on the performance indexes is similar to that of $\kappa_{2}$. However, with high gain selection, the chattering effect around the sliding surface increases. Hence, the gains $\kappa_{1}$ and $\kappa_{2}$ are selected to ensure a good compromise between chattering phenomenon, robustness, and performances.

\section{CONCLUSION}

In this paper, an output feedback active control approach was proposed for the suspension control of a nonlinear quarter car model. An HGO was designed to estimate the states of the nonlinear system and practical stability was established. The controller was designed using the recursive derivative NSTSMbased approach employing a robust STA-based control. To establish robustness of the controller, performance of the controller was evaluated under effect of uncertainties and sensor noise. Based on the performance analysis, it can be deduced that the proposed controller enhanced the ride comfort while ensuring that the road holding was maintained, thus increasing 
the performance of the suspension system. Moreover, an experimental verification of the proposed approach would be an interesting future work.

\section{APPENDIX \\ LIPSCHITZ CONTINUITY FOR $\varphi_{a}, \varphi_{b}$ AND $g\left(x_{3}, U\right)$}

The nonlinear functions $\varphi_{a}$ and $\varphi_{b}$, the states $x_{1}, x_{2}$, and $x_{3}$ are BIBS stable within the operating limits as per Assumption 1 and, hence, bounded. The Lipschitz continuity of the nonlinear function $\varphi_{a}$, thus, depends on $\varphi$, i.e., the suspension nonlinearity. From (3), it can be seen that $\varphi$ is differentiable everywhere except at $x_{2}=0$. Consequently, the Lipschitz constants for the nonlinear function $\varphi_{a}$ can be computed as discussed in [35]. On a similar analogy, with BIBS stability for the states of the system (9), the Lipschitz constants for the nonlinear function $\varphi_{b}$ can be easily computed as $l_{p 3}$ and $l_{p 4}$. The differentiation of the input nonlinearity $g\left(x_{3}, U\right)$ can then be given [41] as

$$
\begin{aligned}
\dot{g}\left(x_{3}, U\right)= & \frac{\lambda}{2 \sqrt{M \rho A_{p}\left(P_{s}-\operatorname{sign}(U) \frac{x_{3}}{M A_{p}}\right)}} U \\
& +\frac{\lambda}{2 \sqrt{M \rho A_{p}\left(P_{s}-\operatorname{sign}(U) \frac{x_{3}}{M A_{p}}\right)}} \operatorname{sign}(\dot{U}) .
\end{aligned}
$$

It can be seen that due to the presence of the $\operatorname{sign}(U)$ function, the above-mentioned expression of $g\left(x_{3}, U\right)$ is not differentiable when $U=0$. Typically, when the magnitude of the supply pressure is equivalent to the pressure difference across the actuator, i.e., when $P_{s}=\frac{x_{3}}{M A_{n}}$, the Lipschitz property of the nonlinearity is lost. Based on the differentiability of this nonlinearity [41], we can further establish that this nonlinearity is differentiable everywhere except at $U=0$. Thus, the local Lipschitz property of the nonlinearity $g\left(x_{3}, U\right)$ can be computed as $l_{p 5}$ similar to [27], [41].

\section{REFERENCES}

[1] H. Li, X. Jing, and H. Karimi, "Output feedback based H-infinity control for vehicle suspension systems with control delay," IEEE Trans. Ind. Electron., vol. 61, no. 1, pp. 436-446, Jan. 2014.

[2] W. Sun, H. Gao, and B. Yao, "Adaptive robust vibration control of full-car active suspensions with electrohydraulic actuators," IEEE Trans. Control Syst. Technol., vol. 21, no. 6, pp. 2417-2422, Nov. 2013.

[3] ABC/MBC Module. (2009). [Online]. Available: http://500sec.com/ $? \mathrm{~s}=$ magic $+\% 20 \mathrm{body}+$ control

[4] M. Strassberger and J. Guldner, "BMW's dynamic drive: An active stabilizer bar system," IEEE Control. Syst. Mag., vol. 24, no. 4, pp. 28-29, Aug. 2004.

[5] W. D. Jones, "Easy ride: Bose Corp. uses speaker technology to give cars adaptive suspension," IEEE Spectr., vol. 42, no. 5, pp. 12-14, May 2005.

[6] M. Moradi and A. Fekih, "Adaptive PID-sliding mode fault tolerant control approach for vehicle suspension systems subject to actuator faults," IEEE Trans. Veh. Technol., vol. 63, no. 3, pp. 1041-1054, Mar. 2014.

[7] M. M. Ma and H. Chen, "Disturbance attenuation control of active suspension with non-linear actuator dynamics," IET Control Theory Appl., vol. 5, no. 1, pp. 112-122, Jan. 2011.

[8] W. Sun, Z. Zhao, and H. Gao, "Saturated adaptive robust control for active suspension systems," IEEE Trans. Ind. Electron., vol. 60, no. 9, pp. 3889-3896, Sep. 2013.

[9] L. Xiao and Y. Zhu, "Sliding-mode output feedback control for active suspension with nonlinear actuator dynamics," J. Vib. Control, vol 20, no. 9, pp. 1-18, Dec. 2013.
[10] H. Li, J. Yu, C. Hilton, and H. Liu, "Adaptive sliding-mode control for nonlinear active suspension vehicle systems using T-S fuzzy approach," IEEE Trans. Ind. Electron., vol. 60, no. 8, pp. 3328-3338, Aug. 2013.

[11] Y. Zhang and A. Alleyne, "A practical and effective approach to active suspension control,"Veh. Syst. Dyn., vol. 43, no. 5, pp. 305-330, Feb. 2005.

[12] S. Yin and Z. Huang, "Performance monitoring for vehicle suspension system via fuzzy positivistic C-means clustering based on accelerometer measurements," IEEE/ASME Trans. Mechatronics, vol. 20, no. 5, pp. 2613-2620, Oct. 2015

[13] B. L. J. Gysen, J. J. H. Paulides, J. L. G. Janssen, and E. A. Lomonova, "Active elctromagnetic suspension system for improved vehicle dynamics," IEEE Trans. Veh. Technol., vol. 59, no. 3, pp. 1156-1163, Mar. 2010.

[14] W. Sun, H. Gao and O. Kaynak, "Adaptive backstepping control for active suspension systems with hard constraints," IEEE/ASME Trans. Mechatronics, vol. 18, no. 3, pp. 1072-1079, Jun. 2012.

[15] J. Zhou and C. Wen, Adaptive Backstepping Control of Uncertain Systems, Berlin, Germany: Springer Verlag, Jan. 2008.

[16] J. Hu, Y. Qiu, and L. Liu, "High-order sliding-mode observer based output feedback adaptive robust control of a launching platform with backstepping," Int. J. Control, to be published, doi: 10.1080/ 00207179.2016 .1147604$.

[17] H. Li, J. Wang, and P. Shi, "Output feedback based sliding mode control for fuzzy systems with actuator saturation," IEEE Trans. Fuzzy Syst., to be published, doi: 10.1109/TFUZZ.2015.2513085.

[18] L. Fridman, J. A. Moreno, and R. Iriate, Sliding Modes After the First Decade of the 21st Century: State of the Art, New York, NY, USA: Springer-Verlag, Sep. 2011.

[19] Y. Shtessel, M. Taleb, and F. Plestan, "A novel adaptive-gain supertwisting sliding mode controller: Methodology and application," Automatica, vol. 48, no. 5, pp. 759-769, May. 2012.

[20] H. Rios, J. Davila, and L. Fridman, "High-order sliding mode observers for nonlinear autonomous switched systems with unknown inputs," J. Franklin Inst., vol. 349, no. 10, pp. 2975-3002, Dec. 2012.

[21] K. C. Veluvolu and Y. C. Soh, "Multiple sliding mode observers and unknown input estimations for Lipschitz nonlinear systems," Int. J. Robust Nonlinear Control, vol. 21, no. 11, pp. 1322-1340, Jul. 2011.

[22] K. C. Veluvolu and D. Lee, "Sliding mode high-gain observers for a class of uncertain nonlinear systems," Appl. Math. Lett., vol. 24, no. 3, pp. 329-334, Oct. 2011

[23] K. C. Veluvolu, Y. C. Soh, W. Cao, and Z. Y. Liu, "Observer with multiple sliding mode for a class of nonlinear uncertain systems," in Proc. 2015 Amer. Control Conf., Portland, OR, USA, Jun. 2005, pp. 2445-2450.

[24] H. Li, H. Gao, P. Shi, and X. Zhao, "Fault tolerant Markovian jump stochastic systems via the augmented sliding mode observer approach," Automatica, vol. 50, no. 7, pp. 1825-1834, Jul. 2014.

[25] Y. M. Sam, J. H. S. Osman, and M. R. A. Ghani, "A class of proportionalintegral sliding mode control with application to active suspension system," Syst. Control Lett., vol. 51, pp. 217-223, Mar. 2004.

[26] S. Yin, X. Zhu, J. Qiu, and H. Gao,"State estimation in nonlinear system using sequential evolutionary filter," IEEE Trans. Ind. Electron., vol. 63, no. 6, pp. 3786-3794, Jun. 2016.

[27] J. Yao, Z. Jiao, and D. Ma, "Extended-state-observer-based output feedback nonlinear robust control of hydraulic systems with backstepping," IEEE Trans. Ind. Electron., vol. 61, no. 11, pp. 6285-6293, Nov. 2014.

[28] K. C. Veluvolu, Y. C. Soh, W. Cao, and Z. Y. Liu, "High gain observer with sliding mode for nonlinear state estimation and fault reconstruction," J. Franklin Inst., vol. 351, no. 4, pp. 1995-2014, Apr. 2014.

[29] K. C. Veluvolu and Y. C. Soh, "High gain observers with sliding mode for state and unknown input estimations," IEEE Trans. Ind. Electron., vol. 56, no. 9, pp. 3386-3393, Sep. 2009.

[30] J. J. Rath, K. C. Veluvolu, and M. Defoort, "Output feedback based sliding mode control of active suspension using backstepping," in Proc. 3rd Int. Conf. Control Eng. Inf. Technol., May 2015, pp. 1-6.

[31] J. Davila, L. Fridman, and A. Levant, "Second-order sliding mode observer for mechanical systems," IEEE Trans. Automat. Control, vol. 50, no. 11, pp. 1785-1789, Nov. 2005.

[32] J. A. Moreno and M. Osorio, "Strict Lyapunov functions for the supertwisting algorithm," IEEE Trans. Automat. Control, vol. 57, no. 4, pp. 1035-1040, Apr. 2012.

[33] C. S. Chiu, "Derivative and integral terminal sliding mode control for a class of MIMO nonlinear systems," Automatica, vol. 48, no. 2, pp. 316-326, Feb. 2012.

[34] R. Rajamani and J. Hedrick, "Adaptive observers for active automotive suspensions: theory and experiment," IEEE Trans. Control Syst. Technol., vol. 3, no. 1, pp. 86-93, Mar. 1995. 
[35] J. J. Rath, K. C. Veluvolu, and M. Defoort, "Simultaneous estimation of road profile and tyre road friction for automotive vehicle," IEEE Trans. Veh. Technol., vol. 64, no. 10, pp. 4461-4471, Oct. 2015.

[36] J. Hahn, R. Rajamani, and L. Alexander, "GPS based real time identification of tire-road friction coefficient," IEEE Trans. Control Syst. Technol., vol. 10, no. 3, pp. 331-343, May 2002.

[37] G. Koch and T. Kloiber, "Driving state adaptive control of an active vehicle suspension system," IEEE Trans. Control Syst. Technol., vol. 22, no. 1, pp. 44-57, Jan. 2014.

[38] Q. Zheng, L. Q. Gao, and Z. Gao, "On stability analysis of active disturbance rejection control for nonlinear time varying plants with unknown dynamics," in Proc. 46th IEEE Conf. Decision Control, Dec. 2007, pp. 3501-3506.

[39] A. Levant, "Higher order sliding modes, differentiation and outputfeedback control," Int. J. Control., vol. 79, no. 9/10, pp. 924-941, Nov. 2003.

[40] W. Sun, H. Gao, and O. Kaynak, "Vibration isolation for active suspensions with performance constraints and actuator saturation," IEEE/ASME Trans. Mechatronics, vol. 20, no. 2, pp. 675-683, Apr. 2015.

[41] B. Yao, F. Bu, J. Reedy, and George T. C. Chiu, "Adaptive robust motion control of single road hydraulic actuators: Theory and experiments," IEEE/ASME Trans. Mechatronics, vol. 5, no. 1, pp. 79-91, Mar. 2000.

[42] D. Traore, F. Plestan, A. Glumineau, and J. D. Leon, "Sensorless induction motor: High order sliding mode controller and adaptive interconnected observer," IEEE Trans. Ind. Electron., vol. 55, no. 11, pp. 3818-3827, Nov. 2008

[43] R. Findeisen, L. Imsland, F. Allgower, and B. Foss, "Output feedback stabilization of constrained systems with nonlinear predictive control," Int. J. Robust Nonlinear Control, vol. 13, pp. 211-227, Feb. 2003.

[44] A. N. Atassi and H. K. Khalil, "A separation principle for the stabilization of a class of nonlinear systems," IEEE Trans. Automat. Control, vol. 44, no. 9, pp. 1672-1687, Sep. 1999. 